\title{
DETERMINATION OF ANTIGEN LEVELS: T-PLASTIN, TRANSGELIN AND CA-125 IN BABOONS WITH INDUCED ENDOMETRIOSIS
}

\author{
Ann Rose Githinji ${ }^{1 *}$, Kenneth Ogila, Mutinda C. Kyama. ${ }^{2}$, Vicky Gent ${ }^{1}$, Erastus Mwadondo ${ }^{1}$, Nyachieo Atunga $^{3}$, \\ Daniel C. Chai $^{3}$, Rebecca Waihenya ${ }^{1}$ \\ ${ }^{1}$ Department of Zoology, College of Pure and Applied Sciences, Jomo Kenyatta University of Agriculture and \\ Technology \\ P.O Box 62000-00200, Nairobi, Kenya \\ *agithinji@jkuat.ac.ke \\ ${ }^{2}$ Department of Medical laboratory Sciences, College of Health Sciences, Jomo Kenyatta University of \\ Agriculture and Technology \\ P.O Box 62000-00200, Nairobi, Kenya \\ ${ }^{3}$ Reproduction Health and Biology, Institute of Primate Research. \\ P.O. Box 24481 Karen 00502, Kenya
}

\begin{abstract}
Endometriosis is defined as the presence of endometrial-like tissue outside the uterine cavity and is associated with chronic intra-pelvic inflammation. Its symptoms, which are often severe, have a negative impact on a woman's quality of life. They include chronic pelvic pain and infertility. It is estimated that about $10 \%$ women in their reproductive ages are affected by the disease globally. Currently, diagnostic methods are laparoscopy with histological confirmation and non-invasive such as ultrasound and the quantification of CA-125 biomarker in serum. CA-125 is known to be elevated in endometriosis, however it does not have sufficient diagnostic power as a single biomarker of endometriosis. Due to this, there is a need to determine other antigens that would act as potential biomarkers for endometriosis. It has been observed that Transgelin and T-Plastin are upregulated in endometriosis lesions and in secretory phase of endometrium respectively. There is insufficient data available showing the concentration of T-plastin in serum or plasma in the development of endometriosis. The main objective of this study was to identify whether T-plastin, Transgelin and CA-125 are potential biomarkers in early diagnosis of endometriosis using animal model for endometriosis. The baboon model represents clinically relevant research models for endometriosis. This study compared the levels of T-plastin, Transgelin and CA-125 in the peripheral blood and peritoneal fluid of baboons before and after induction of endometriosis. In this prospective, case-control study, ten female baboons (Papio anubis, 9-15 kg each) of proven fertility that have had at least one menstruation during captivity, were induced by intra-pelvic injection of menstrual endometrium on day 1 or 2 of menstruation followed by staging laparoscopy. Serum and peritoneal fluid samples were collected prior to induction to serve as controls.T-Plastin, Transgelin and CA-125 were measured using commercially available ELISA Kits. Data was analyzed using non-parametric test and level of significance at $\mathrm{p}<0.005$. T-Plastin and Transgelin had insignificant $\mathrm{p}$ values in both peripheral blood and peritoneal fluid while CA-125 showed $\mathrm{p}=0.0003$ in peripheral blood and $\mathrm{p}=0.0279$ in peritoneal fluid. Diagnostic performance of individual biomarkers was determined by Receiver Operating Characteristic (ROC) with the Area under the curve (AUC) equal to 0.5 showing T-Plastin and Transgelin in both serum and peritoneal fluid unreliable for use as biomarkers in endometriosis. CA125 levels in peritoneal fluid had an AUC of 0.7900 indicating it can be used as potential biomarkers for endometriosis.
\end{abstract}

Key words: Endometriosis, biomarkers, T-Plastin, Transgelin, CA-125, ELISA, Olive Baboons.

DOI: $10.7176 / A L S T / 75-06$

Publication date:August $31^{\text {st }} 2019$

\section{Introduction}

Endometriosis is a common benign, chronic and multifactorial complex gynecological disease which is estrogen dependent (Sinaii et al., 2008; D'Hooghe, 1997). It is characterized by chronic pelvic pain, dysmenorrhea and infertility and defined by presence of endometrial stroma and glands outside the uterine cavity (Nisoile \& Donnez, 1997; Farquhar, 2001). Clinically endometriosis presents in three varying phenotypes; peritoneal endometriosis, ovarian endometriotic cyst and deep infiltrating endometriosis (D'Hooghe et al., 2003). Ten percent of women in the reproductive age globally are reported to suffer from endometriosis (Olive \& Schwartz, 1993; Cramer \& 
Wilson, 1987; Giudice \& Kao, 2004), while 40-60\% of women with infertility and chronic pelvic pain have endometriosis (Vermeuleri et al., 2014). According to American Society of Reproductive Medicine (ASRM, 1996) report on classification and staging of endometriosis, there are four classes/stages of endometriosis, minimum, mild, moderate and severe (1-4 stages). There is no correlation between the symptoms and the stage of the disease (Mehedintu et al., 2014). Despite the studies done on endometriosis, its etiology remains unclear (Baldi et al., 2008). Several theories have been hypothesized; the most popular and well accepted being retrograde menstruation in which endometrium fragments are refluxed via fallopian tubes into the pelvis (Sampson, 1927). Inflammatory factors such as IL-1, IL-2, IL-6, IL-8, and TNF alpha (expressed by macrophages) are associated to developmental of endometriosis (Ioana \& Razoan, 2013). A defective immunosurveillance against endometrial cells deposited on the peritoneal cavity due to a dysfunctional immune system in women allows development of endometriosis (Leboric et al., 2001). Due to lack of a non-invasive diagnostic test, there is usually a delay of 8-11 years (Fassbender et al., 2015; Fauconnier \& Charpron, 2000). Endometriosis is sometimes asymptomatic (Rawson, 1991). Studies have shown that levels of Transgelin increase in lesions in endometriosis (Kyama et al., 2006), while T-Plastin is upregulated in the secretory phase in endometriosis (Kyama et al., 2011). CA125 elevated in endometriosis is used as a serum marker in endometriosis (Chen et al., 1998; Barbati et al., 1994). The gold standard for diagnosis is laparoscopy followed by histological confirmation (Kennedy et al., 2005). A simple, lowcost, non-invasive, fast, accurate and reliable diagnostic test will solve the delay and give light to treatment at early stages of the disease.

In this study, ten female baboons were induced with endometriosis. Peripheral blood and peritoneal fluid samples were collected at three stages, prior to induction, twenty six days after induction and upon full development of endometriosis. Enzyme Linked Immunosorbent Assay (ELISA) was used to evaluate the antigen levels. The study sought to determine the T-plastin, Transgelin and CA125 antigen levels in peripheral blood and peritoneal fluid using ten baboons induced with endometriosis and evaluate their variability as potential biomarkers for the detection of the onset of endometriosis.

\section{$2 \quad$ Materials and Methods}

\subsection{Study site}

The study was carried out at Institute of Primate Research (IPR), Karen, Nairobi, Kenya where the animals were maintained. Laboratory analysis were carried out from the Reproductive Health Laboratory of IPR and Biochemistry Department of Kenyatta National Hospital (KNH).

\subsection{Study design and subjects}

The research design was prospective, case-control study of ten female baboons (Papio anubis). The animals weighed approximately $9-15 \mathrm{~kg}$ and were of proven fertility, that is, they had at least one menstruation during captivity. All the animals were obtained from the Institute of Primate Research (IPR) in Karen. Care of the animals and all experimental procedures were approved by the Institutional Animal Care and Use Committee.

The animals were housed indoors in well-lit as natural as possible environment and were fed with commercial pellets and substitute of vegetables and fruits.

\subsection{Induction of endometriosis}

Induction of endometriosis was performed in ten baboons after establishment of a normal pelvis (screening laparoscopy 1) on the $1^{\text {st }}$ or $2^{\text {nd }}$ day of menstruation (screening laparoscopy 2, cycle 2), by intrapelvic injection (D'Hooghe et al., 1995) followed by staging laparoscopy (laparoscopy 3, cycle 3).

Endometrial tissue was extracted from each baboon by transcervical curettage, and fragmented through an 18gauge needle creating a menstrual "paste" as previously described (D'Hooghe et al., 1995; Chai et al., 2007). A standardized amount of the resulting paste $(1000 \mathrm{mg})$ of tissue paste was then seeded onto various peritoneal sites (uterosacral ligaments, uterovesical folds, posterior uterine peritoneum, cul-de-sac, broad ligament and ovary) as described before (D'Hooghe et al., 1995).

\subsection{Collection of Peripheral Blood (PB)}

Four milliliters of venous blood was collected from each baboon. Blood collection was carried out prior to induction, $26^{\text {th }}$ and $60^{\text {th }}$ day post induction. . Following blood collection, it was gently inverted several times (approx.7-10 times) to prevent the formation of blood clot. The peripheral blood samples were then centrifuged at 
$3000 \mathrm{rpm}$ for 10 minutes at $4^{\circ} \mathrm{C} .500 \mu \mathrm{l}$ aliquots of plasma was prepared into labeled tubes and stored at $-80^{\circ} \mathrm{C}$ until use.

\subsection{Collection of Peritoneal Fluid}

Peritoneal fluid was aspirated using a $20 \mathrm{cc}$ syringe, this was done prior to induction, $25^{\text {th }}$ and $60^{\text {th }}$ day post induction. Following Peritoneal Fluid (PF) collection, the volume was measured and color appearance recorded. The PF samples were centrifuged at $1500 \mathrm{rpm}$ for 10 minutes. $500 \mu 1$ aliquots of PF supernatant were prepared into labeled tubes and stored at $-80^{\circ} \mathrm{C}$ until use.

\subsection{Enzyme Linked Immunosorbent Assay}

T-Plastin, Transgelin and CA-125 were evaluated on blood and peritoneal samples before and after induction of endometriosis using ELISA according to manufactures' instructions. Levels of T-Plastin were evaluated using Human Plastin 3 (PLS3) ELISA kit (My BioSource, USA, Cat. No. MBS9312912). Briefly, the serum and peritoneal fluid samples were brought to room temperature prior to starting the assay procedure. Fifty microliters of the samples and sample diluent were added to each well of the 96 well ELISA plate, in duplicate. Horse radish Peroxidase conjugate was added to each well at a quantity of $100 \mu 1$. The plates were incubated for 60 minutes at a temperature of $37^{\circ} \mathrm{C}$. The plates were then washed four times with wash buffer. Chromogen solutions were added and the plates were placed in the dark for 15 minutes at $37^{\circ} \mathrm{C}$ to allow color formation. Fifty microliters of Stop solution was added to the wells. The Optical Density was read at 430nm using the ELISA reader (Biotek Elx 808).

Transgelin levels were evaluated using the Transgelin (TAGLN) ELISA Kit (online.com antibodies, Germany, Cat. No. ABIN 1567594). Serum and peritoneal fluid samples were brought to room temperature prior to running the assay. A hundred microliters of the sample was added to the wells and incubated for ninety minutes at $37^{\circ} \mathrm{C}$. Biotinylated detection antibody was then added into each well and incubated for an hour at $37^{\circ} \mathrm{C}$ before washing thrice with a wash buffer $(350 \mu \mathrm{l}) .100 \mu \mathrm{l}$ of Horse radish Peroxidase conjugate was added into each well and incubated for thirty minutes at $37^{\circ} \mathrm{C}$. The plates were washed with $350 \mu \mathrm{l}$ of buffer five times before a ninety microliter substrate solution was added, incubated for fifteen minutes and the reaction stopped with fifty microliters of stop solution. Optical density measurements were determined using Biotek Elx 808 ELISA machine and a micro plate reader set at $450 \mathrm{~nm}$.

One milliliter of each serum and peritoneal fluid samples were brought to room temperature. The vials containing the samples were fed into Cobas E601 automated analyzer of Cobas 6000 series (Roche Diagnostics) to determine the CA-125 levels.

\subsection{Statistical Analysis}

Data was analyzed using non parametric test, Mann Whitney. The statistical level of significance was set at $\mathrm{p} \leq$ 0.05. This was done using the GraphPad Prism software version 7.00 for Windows. Evaluation of the diagnostic performance of the tested biomarkers was performed by calculating the area under curve (AUC) of Receiver Operating Characteristics (ROC) curves. AUC of 0.5 and below indicated the test was positive $50 \%$ of the time.

\subsection{Ethical Consideration}

The experimental protocols carried out in this study underwent review by the Institutional Science and Ethics Committee (ISERC) based at IPR, Kenya.

\section{Results}

\subsection{Levels of T-plastin, Transgelin and CA125 biomarkers in serum}

A total of ten baboons induced with endometriosis were evaluated for $\mathrm{T}$ plastin, Transgelin and CA-125 concentrations in plasma. There was a slight increase in levels of T-plastin 30 days after induction, however this was not significant ( $\mathrm{p}=0.8633$ ). After 30 days the levels again increased slightly, however this was not significant $(p=0.9999)$ (Fig 1). Transgelin showed insignificant increase in concentration after first post-induction. There was slight increase in concentration after the $60^{\text {th }}$ post induction, however this difference was not significant. CA-125 concentrations in plasma showed significant increase 30 days after induction $(\mathrm{p}=0.0003)$. There was also a significant rise in concentration of CA-125 after the $60^{\text {th }}$ day post -induction $(p=0.0014)$. 


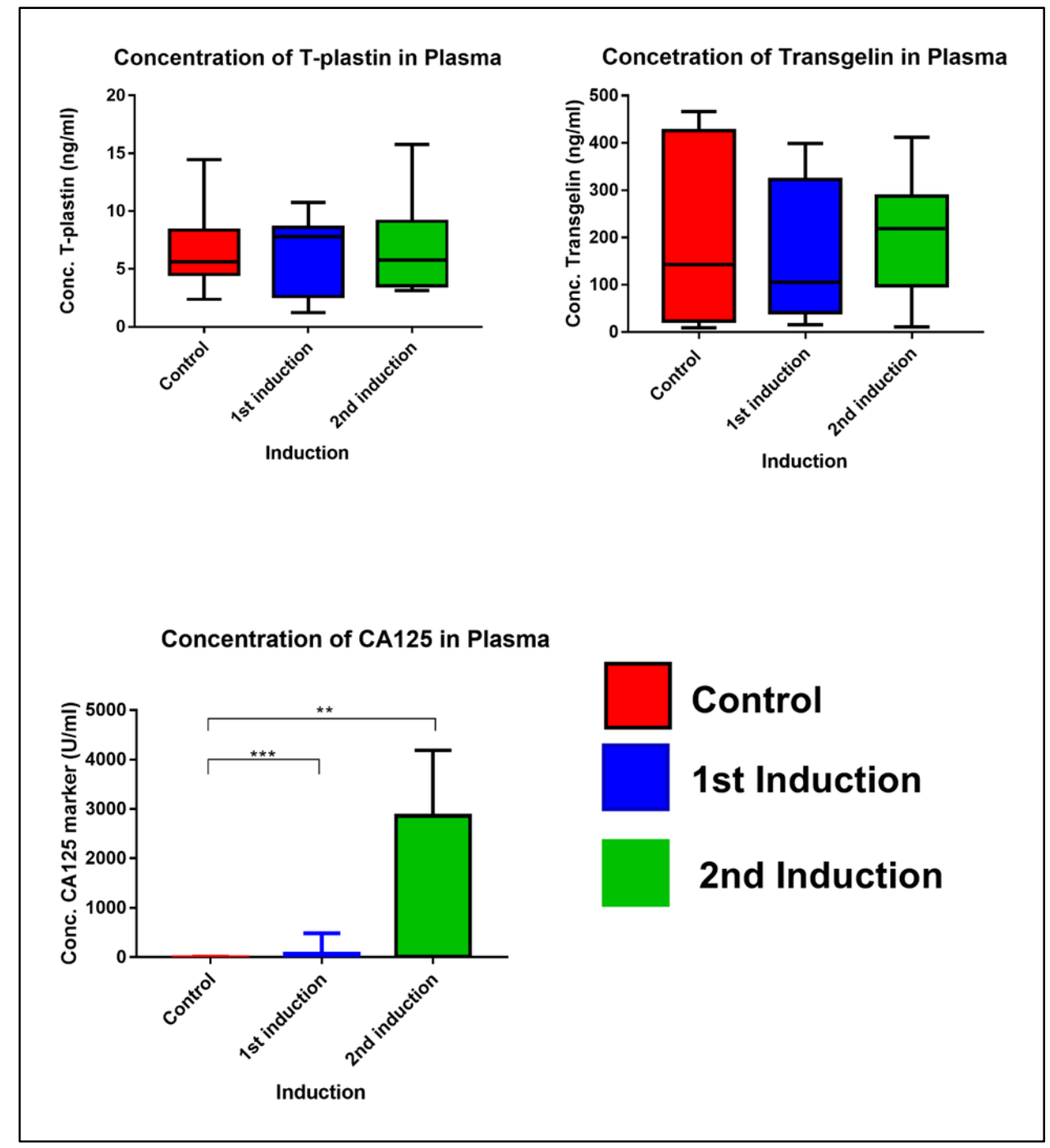

Fig 1: Concentrations of T-plastin, Transgelin and CA125 Pre-induction, $1^{\text {st }}$ post-induction and $2^{\text {nd }}$ post-induction in peripheral blood in baboons induced with endometriosis. Values were considered significant when $\mathrm{p} \leq 0.05$ and assigned $* * \mathrm{p}=0.0014, * * * \mathrm{p}=0.0003$.

\subsection{Levels of biomarkers in peritoneal fluid.}

Ten baboons induced with endometriosis were evaluated for the concentrations of T-plastin, Transgelin and CA125 in peritoneal fluid. Figure 2 shows the concentration of T-plastin observed with a slight increase between pre induction and both post induction measurements. The increase however was not significant $(\mathrm{p}=0.4463)$. Transgelin did not show significant rise in concentration between pre and the post induction readings in peritoneal fluid with $\mathrm{p}=0.1192$. The results also showed that CA 125 concentrations were significantly higher in peritoneal fluid especially between pre induction and $1^{\text {st }}$ post induction reading $(\mathrm{p}=0.0279)$. The concentration between pre induction and $2^{\text {nd }}$ post induction however was insignificant, $(\mathrm{p}=0.9682)$. 


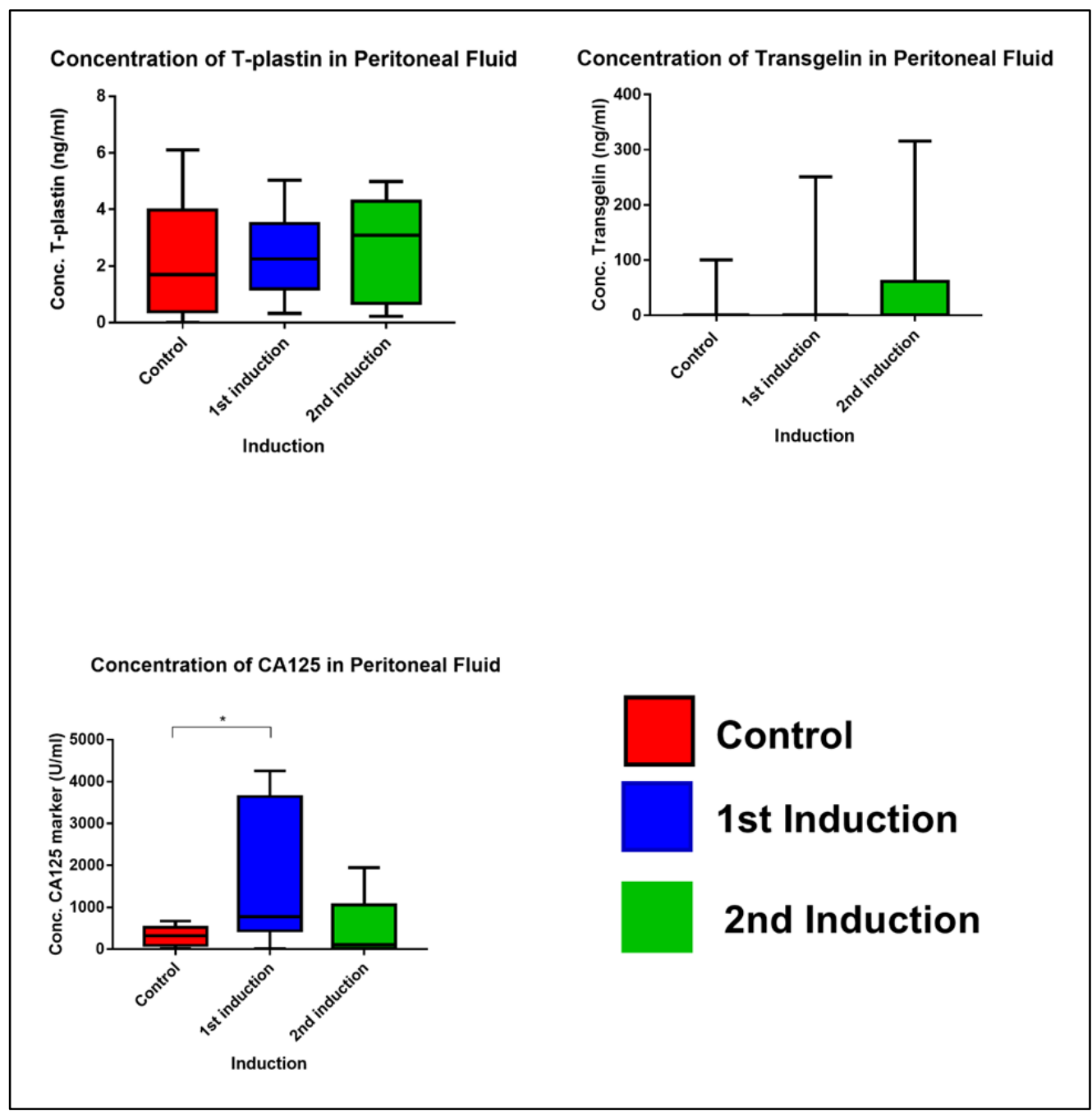

Fig 2: Concentrations of T-Plastin, Transgelin and CA-125 pre-induction, 1 st and $2^{\text {nd }}$ post-induction in peritoneal fluid of baboons induced with endometriosis. Values were considered significant when $p \leq 0.05$ and assigned $* p=0.0279$

\subsection{Receiver operating Curves (ROC) Analysis}

Analysis determining the diagnostic performance of the biomarkers in plasma using the receiver operating characteristics (ROC) showed T-plastin to have an area under curve AUC $=0.5$, followed by Transgelin that had $\mathrm{AUC}=0.505$. CA125 had a markedly higher area under curve $(\mathrm{AUC}=0.79)$, as shown in figure 3. 

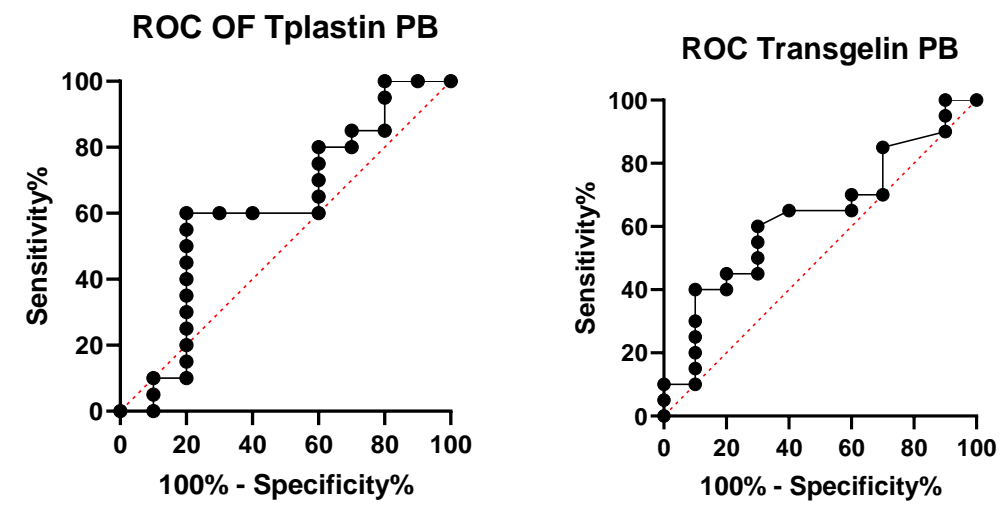

ROC OF CA125 PB

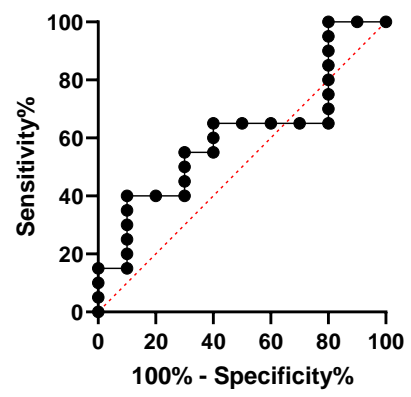

Fig 3: Receiver operating characteristics (ROC) curves of T-plastin, Transgelin and CA-125 in plasma. T-plastin$A U C=0.5$ with 95\% confidence interval (CI) of 0.2275-0.7727, $p>0.999$, Transgelin, AUC $=0.505$ with $95 \% C I$ $0.2455-0.764, p=0.9698, C A-125$ has $A U C=0.6100,95 \%$ CI 0.395-0.8200, $p$ value $=0.3331$

The receiver operating characteristics (ROC) for concentrations of T-plastin, Transgelin and CA-125 in peritoneal fluid showed an area under curve $\mathrm{AUC}=0.6325$ for $\mathrm{T}$ plastin, $\mathrm{AUC}=0.615$ for Transgelin while CA125 had a markedly high area under curve, AUC $=0.7900$, as shown in figure 4 .

ROC of Tplastin PF

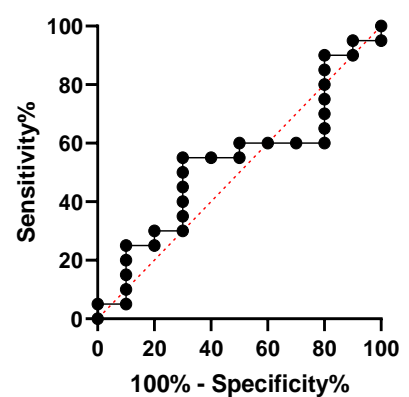

Transgelin PF

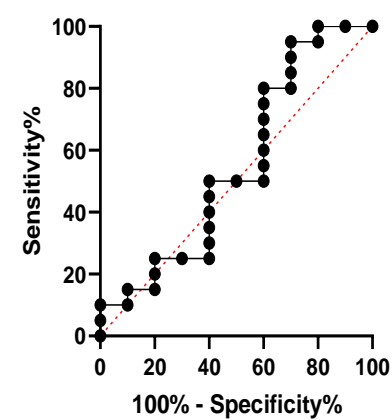

ROC OF CA125 PF

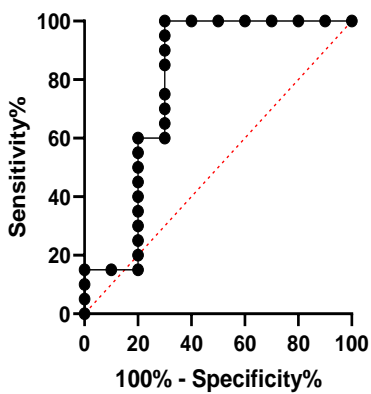

Fig 4: Receiver operating characteristics (ROC) curves of T-plastin, Transgelin, and CA-125 in peritoneal fluidPlastin-AUC $=0.6125$ with $95 \%$ CI 0.3242-0.9008, $p=0.4239$, Transgelin has AUC $=0.685$ with $95 \%$ CI 0.44220.9278 and a $p$ value $=0.1620$. CA125 AUC $=0.7900$ with 95\% CI 0.5723-1.000 $p=0.0107$

\section{Discussion}

The baboons developed endometriosis within the two month period after induction. Samples of blood and peritoneal fluid collected prior and post induction were analyzed for T-plastin, Transgelin and CA-125 concentrations both by ELISA and non-parametric analysis. The results indicate that concentrations of T-Plastin and Transgelin in serum were detected and measured but no significant increase was realized during the two months of study between the pre and the post induction samples. Slight increase was noted in T-plastin between pre induction and endometriosis group. Transgelin appeared to have higher concentration in serum prior to induction and only slight increase was observed after induction. A study by Hidalgo et al., 2011, found that Transgelin is increased in endometriotic lesions compared with eutopic endometrium. The study suggested Transgelin may be associated with development and maintenance of endometriosis. The area under curve (AUC) in receiver operating characteristics (ROC) that was used to determine diagnostic performance was quite low for the biomarkers ( $\mathrm{AUC}=0.535, \mathrm{AUC}=0.550$ ) respectively indicating the two biomarkers in serum cannot be effective or suitable test for endometriosis. The endometriosis established during the two months study is likely to have been minimum-mild stages where antigens are detectable but not significantly increased. Serum CA-125 was seen to 
have a significant rise $(\mathrm{p}=0.0003$ and $\mathrm{p}=0.0014)$ between pre induction, $1^{\text {st }}$ post induction and $2^{\text {nd }}$ post induction. It has been established that CA-125 increases in initial stages of endometriosis but markedly increases in moderate to severe stages (Muyldermans et al., 1995; Mol et al., 1998). Another study indicates CA-125 test has a specificity of $89 \%$ in detecting moderate to severe endometriosis (Child \& Tan, 2001). T-Plastin which has been observed to regulate actin assembly and cellular motility was upregulated, however this has not been quantified in proteomics studies. T-plastin plays a major role in development of abnormal structures in pelvic peritoneum in endometriosis (Kyama et al., 2011). However little information regarding presence of T-plastin in peripheral blood during the development of endometriosis is available.

Transgelin which is believed to be related to cell differentiation, cell migration and involved in tissue invasion was remarkably upregulated in a study by Kyama et al., 2006 and Tawfik et al., 2014) indicated Transgelin to be a specific marker of smooth muscle differentiation and capable of being used as a useful marker in addition to other antigens. Significant rise in concentrations was expected in endometriosis.

CA-125 levels increase in serum in moderate to severe endometriosis, Bon et al., 1995. The study indicates that CA-125 released from endometrial cells can gain access to circulation and lymphatic system. CA-125 was significantly elevated in a study by Patton et al., 1986. Another study by Szubert et al., 2012 had similar observations, CA-125 levels in patients with endometriosis in moderate to severe stages was significantly raised. Similar results of an increase in CA-125 levels have been found by Giudice et al., 1986.

The study of T-plastin and Transgelin concentrations in peritoneal fluid did not reveal any significant difference between the control and endometriosis. This is due to the mild -minimum stages of endometriosis in the two months it was developed. The concentration of CA-125 however was seen to have significantly increased between the pre induction and disease in the peritoneal fluid ( $\mathrm{P}$ value $<0.005)$. This is due to $\mathrm{CA}-125$ and other proteins such as glycodelins being released into peritoneal cavity after secretion by the endometrial cells and finding their way into peritoneal cavity by retrograde menses.

In a study by Szubert et al., 2012, serum CA-125 levels were significantly higher in endometriosis compared to controls. Peritoneal fluid CA-215 was also seen to be higher in disease than in controls (Amaral et al., 2006). This is a result of the presence of blood and eutopic endometrial tissue into the peritoneal cavity due to retrograde menses. Bon et al., 1995 has also suggested that CA-125 released from the endometrium can have access to the lymphatics and the circulation. CA-125 plays a role in development of endometriosis.

The major limitation observed in this study was the animals developed endometriosis for two months only and therefore moderate to severe stages were not achieved. Different assays, such as Western blot, flow cytometry, immunohistochemistry, would be more sensitive and detect the increase in concentrations of these antigens in serum.

\section{Conclusion}

This study reveals presence of T Plastin, Transgelin and CA-125 in serum and peritoneal fluid. There was no significant increase in $\mathrm{T}$ plastin and Transgelin concentrations between the pre induction and post induction and therefore cannot be an accurate test or reliable biomarker for endometriosis.CA-125 levels increased both in serum and peritoneal fluid. However, the low diagnostic power does not allow CA-125 to be used as a reliable individual biomarker.

Further work using different protocols would be recommended to identify the tread of these antigens in the minimum to mild stages in endometriosis. Evaluation of the potential of combining the three antigens, CA-125, Transgelin and T-plastin to improve detection of endometriosis in early stages.

Conflict of interest: There is no conflict of interest among authors in this work

\section{Reference}

Amaral, V. F., Ferrlani, R. A. \& SAMF, 2006. Positive Correlation Between Serum and Peritoneal Fluid CA-125 Levels in Women With Pelvic Endometriosis. Sao Panto Med J. 
ASRM, 1996. Revised ASRM Classification. FertilSteril, p. vol 4.

Baldi, A., Camplon, M. \& Signovire, P., 2008. Endometriosis:Pathogenesis,Diagnosis,Therapy and Association with Cancer(Review). Oncology Reports, pp. 843-846.

Barbati, A., V, C. E. \& Spaziani, R., 1994. Serum and Peritoneal Fluid CA-125 Levels in Patients With Endometriosis. Fertil Steril, 61(1), pp. 438-421.

Bon, G., Kenemans, P. \& Dekker'J.J, 1995. Fluctuations in CA125 and CA15-3 Serum Concentrations During Spontenious Ovulatory Cycles. Human Reproduction, pp. 566-570.

Chen, F., Soong, Y., Lee, N. \& Lo, S., 1998. The Use of CA125 as a Markerfor Endometriosis in Patients with Dysmenorrhea for Monitoring Therapy and for Recurrence of Endometriosis. Acta Obstet Gynecol Scand, pp. 665670.

Child, T. J. \& Tan, L. S., 2001. Endometriosis : Aetiology,Pathogenesis and Treatment. Drugs Pubmed, 6(12), pp. 1735-50.

Cramer, D. \& Wilson, E., 1987. Epidemiology of Endometriosis in Adolescents In:Wilson E.A,editor,Endometriosis. New york Alan Liss, pp. 5-8.

D'Hooghe, 1997. Clinical Relevance of the Baboon as a Model for the Study of Endometriosis. Ferti Steril, pp. 613-625.

D'Hooghe, T. et al., 1995. Intrapelvic Injection of Menstrual Endomentrium Causes Endometriosis in Baboons (Papio Cynocephalus and Papio Anubis)). Am J Obstet Gynecol, pp. 125-35.

D'Hooghe, T., Debrook, S., Hill, J. A. \& Meuleman, C., 2003. Endometriosis and Subfertility:Is the Relationship Resolved?. Semin Reprod Med, pp. 243-254.

Farquhar, C. M., 2001. Extracts from the "Clinical Evidence":Endometriosis. BMJ, pp. 1449-1452.

Fassbender, A. et al., 2015. Update of Biomarkers for Detection of Endometriosis. Biomed Research International, p. 14.

Fauconnier \& Charpron, 2000. Endometriosis and Pelvic Pain:Epidemiological Evidence of the Relationship and Implications. Hum Reprod, pp. 595-606.

Giudice, A. J., Pindeda, J. \& Bell, 1986. Serum Levels of CA-125 in Patients with Endometriosis: a Preliminary Report. Fertil Steril, Volume 45, pp. 876-78.

Giudice, L. \& Kao, L., 2004. Endometriosis. Lancet, pp. 1789-99.

Hidalgo, G.S et al., 2011.TAGLN Expression is Deregulated in Endometriosis and may be Involved in Cell Invasion, Migration and Differentiation.Fertil Steril,Volume 96 Issue3 pp 707-3

Ioana, I. \& Razoan, I., 2013. Cytokines and Endometriosis, The Role of Immunological Alterlations. BIOTECHNOLOGY,MOLECULAR BIOLOGY AND NANO MEDICINE, pp. 2330-9326.

kennedy, S. et al., 2005. ESHRE Guidelines for the Diagnosis and Treatment of Endometriosis. Hum Reprod, pp. 2698-2704.

Kyama, C. et al., 2006. Increased Peritoneal and Endometrial Gene Expression of Biologically Relevant Cytokines and Growth Factors during the Menstrual Phase in Women with Endometriosis. Fertil Steril, pp. 1667-75.

Kyama, C. et al., 2011. Evaluation of Endometrial Biomarkers for Semi-invasive Diagnosis of Endometriosis. FertilSteril, pp. 95:1338-1343.

Leboric, D., Mueller, M. \& Taylor, R., 2001. Immunobiology of Endometriosis. Fertil steril, pp. 1-10.

May, K. et al., 2010. Peripheral Biomarkersof Endometriosis:a Systematic Review. Hum.Reprod.update, pp. 65174.

Mehedintu, C., Plotogea, M. N., Lonescu, S. \& Antonovici, M., 2014. Endometriosis Still a Challenge. J Med Life, 7(3), pp. 349-357. 
Mol, B. et al., 1998. The Performance of CA-125 Measurement in The Detection of Endometriosis. Fertility and Sterility, p. Vol $70 \mathrm{No}^{\wedge}$.

Muyldermans, M., Cornillie, F. \& Koninckx, P., 1995. CA125 and Endometriosis. Human Reproduction Update, pp. 173-187.

Nisoile, M. \& Donnez, J., 1997. Endometriosis. Fertil.steril, pp. 585-596.

Olive, D. \& Schwartz, L., 1993. Endometriosis. New Engl J med, p. 1759.

Patton, P. E., Field, C., Harms, R. \& Coulam, C., 1986. CA-125 Levels in Endometriosis. Fertil Steril, Volume 45, p. 770 .

Rawson, J. M., 1991. Prevalence of Endometriosis in Asymptomatic Women. J.Reprod Med, 6(1), pp. 513-5.

Sampson, J. A., 1927. Metastatic or Embolic Endometriosis Due to the Menstrual Dissemination of Endometrial Tissue into the Venous Circulation. The American Journalof Pathology, pp. 93-110.

Sinaii, N. et al., 2008. Differences in Characteristics Among 1000 Women with Endometriosis Based on Extent of Disease. Fertl Steril, pp. 538-545.

Szubert, M., Suzin, J., Wierzbowski, T. \& Kowalczyk-Amico, L., 2012. CA-125 Concentration in Serum and Peritoneal Fluid in Patients With Endometriosis-Preliminary Results. Arch Med Sci, Volume 8, pp. 504-508.

Tawfik, O. et al., 2014. Transgelin,A Novel Marker of Smooth Muscle Differentiation ,Effectively Distinguishes Endometrial Stromal Tumours From Uterine Smooth Muscle Tumours. Int J Gynecol Obstet Reprod Med Res, 1(1), pp. 26-31.

Vermeuleri, N., Ble, B. d. \& Dunselman, G., 2014. Patient Verson of The ESHRE Guidelines on Management of Women With Endometriosis. eshre. 